\title{
A New Look at the So-Called Trammel of Archimedes
}

\section{Tom M. Apostol and Mamikon A. Mnatsakanian}

1. INTRODUCTION. STANDARD TRAMMEL. Figure 1a shows a line segment of fixed length whose ends slide along two perpendicular axes. It can be realized physically as a sliding ladder or as a sliding door moving with its ends on two perpendicular tracks. During the motion, a fixed point on the segment traces an ellipse with one quarter of the ellipse in each quadrant (Figure 1b), so this device is called an ellipsograph, a mechanism for drawing an ellipse.

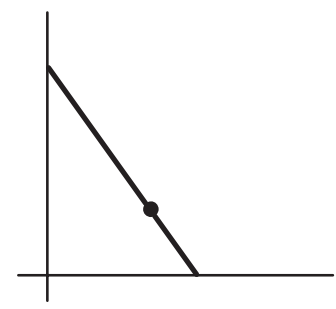

(a)

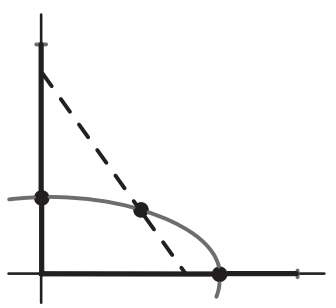

(b)

Figure 1. (a) Standard trammel. Its endpoints move along perpendicular axes. (b) Each point on the trammel traces an ellipse.

This particular ellipsograph was known to ancient Greek geometers and is often called the trammel of Archimedes [6, p. 3], but we are not aware of any historical evidence that suggests who invented it. We refer to it as a standard trammel to distinguish it from two generalizations introduced in this article: a zigzag trammel, obtained by dividing a standard trammel into several hinged pieces (Section 8), and a flexible trammel whose length may vary (Section 9).

The ellipsograph feature alone makes the trammel an important object of study, but it has other interesting properties discovered with methods of calculus that deserve to be better known. This article describes known properties of the standard trammel as well as new ones that can be studied by simple geometric methods that do not require calculus. The generalization to flexible trammels increases our understanding of these properties and also leads to engaging classroom activities.

2. ELLIPSE TRACED BY A POINT ON A STANDARD TRAMMEL. To see why a point on a trammel $A B$ of fixed length $L$ traces an ellipse, we show first that the midpoint $M$ of $A B$ traces a circle, as suggested by Figure 2a. Figure 2b shows segment $O M$, together with perpendiculars from $M$ to each axis, dividing the large right triangle $A O B$ into four congruent smaller right triangles, each having hypotenuse of common length $O M=A M=M B=L / 2$. Therefore, $M$ always lies on a circle with center at $O$ and radius $L / 2$. This also follows by completing the rectangle $O A C B$ in Figure 2c.

The circle of radius $L / 2$ has Cartesian equation

$$
\left(\frac{x}{L / 2}\right)^{2}+\left(\frac{y}{L / 2}\right)^{2}=1 .
$$




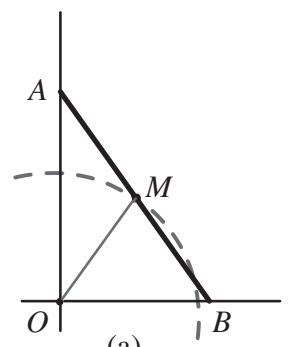

(a)

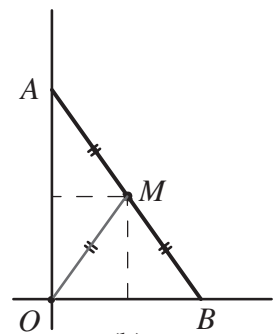

(b)

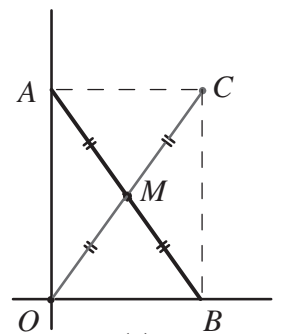

(c)

Figure 2. (a) Midpoint $M$ traces a circle of radius $O M$. (b) Triangle $A O B$ divided into four congruent right triangles. (c) Diagonals of a rectangle are equal and bisect each other.

This is a special case of the general equation of an ellipse,

$$
\left(\frac{x}{a}\right)^{2}+\left(\frac{y}{b}\right)^{2}=1
$$

traced by a point $E=(x, y)$ on the trammel that divides it into segments of lengths $a=A E$ and $b=E B$, as indicated in Figure 3a. We deduce (2) from (1) by relating the coordinates $(x, y)$ of $E$ with the coordinates $(X, Y)$ of the midpoint $M$ which satisfy (1). Similar triangles in Figure 3a reveal that $x / a=X /(L / 2)$ and $y / b=Y /(L / 2)$; hence (2) follows from (1). The same analysis holds if $E$ is any point on the line through the trammel, even if $E$ is outside the trammel as in Figure 3b. In all cases:

The semiaxes of the ellipse are the distances from $E$ to the endpoints $A$ and $B$.

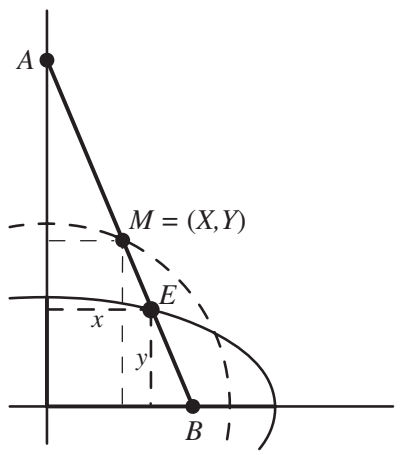

(a)

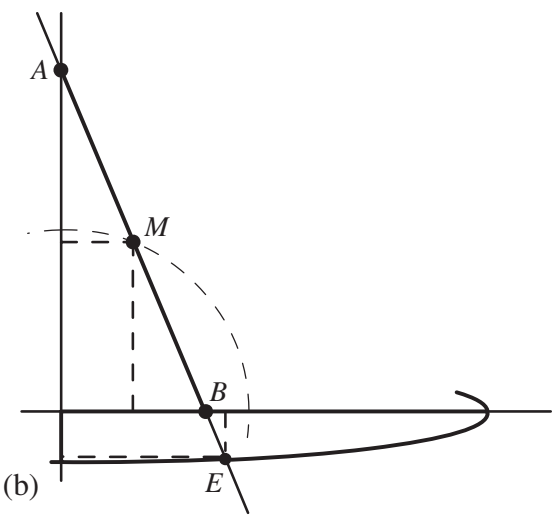

Figure 3. The coordinates $(x, y)$ of $E$ are related to those $(X, Y)$ of midpoint $M$ as follows: $x / a=X /(L / 2)$, and $y / b=Y /(L / 2)$, where $a=A E$ and $b=E B=|L-a|$. In (a), $E$ lies between $A$ and $B$; in (b), $E$ lies outside $A B$ but on the line through $A B$.

3. ASTROID AS THE ENVELOPE OF A STANDARD TRAMMEL. Next we show geometrically that a trammel of length $L$ is always tangent to an astroid, which is defined as the locus of a point $P$ on the circumference of a circle that rolls without slipping inside a larger circle of radius four times as great. Figure $4 \mathrm{a}$ shows the larger circle of radius $L$ centered at the origin, and the smaller circle of radius $L / 4$ rolling inside it. 
Let $C$ denote the point of tangency of the two circles. The midpoint $M$ of $O C$ is on the smaller circle as shown. Triangle $M P C$ is inscribed in a semicircle with diameter $M C$, so angle $M P C$ is a right angle, as indicated. Line $A B$ drawn through $P M$, perpendicular to $C P$, is also tangent to the astroid at $P$ because $C$ is the center of instantaneous rotation of the smaller circle as it rolls inside the larger circle. Therefore, if we show that the length of $A B$ doesn't change as $P$ moves along the astroid, this will show that $A B$ is a trammel. To do this it suffices to show that $M$ is also the midpoint of $A B$, and that triangle $O M A$ is isosceles. Refer to Figure $4 \mathrm{~b}$. The two circular arcs $C L$ and $C P$ have equal lengths because the smaller circle rolls along the larger. Arc $C L$ has length $L \gamma$, where $\gamma$ is the central angle of the larger circle subtending arc $C L$. Similarly, arc $C P$ has length $(L / 4) \beta$, where $\beta$ is the central angle of the smaller circle subtending arc $C P$. The arcs have equal length, so $\beta=4 \gamma$, as indicated in Figure $4 \mathrm{~b}$. But inscribed angle $C M P$ on the smaller circle is half the central angle, or $2 \gamma$. This is also the vertical angle $O M B$. A vertical line through $M$ makes angle $\gamma$ with $O C$, so it bisects angle $C M A$ as shown. Hence triangle $O M A$ is isosceles, with each base angle equal to $\gamma$. This implies that triangle $O M B$ is also isosceles with base angles $\alpha$ complementary to $\gamma$. Therefore $A M=M B=M O=L / 2$, which shows that $A B$ has fixed length $L$, so it is a standard trammel, and we have already seen that it is always tangent to the astroid.

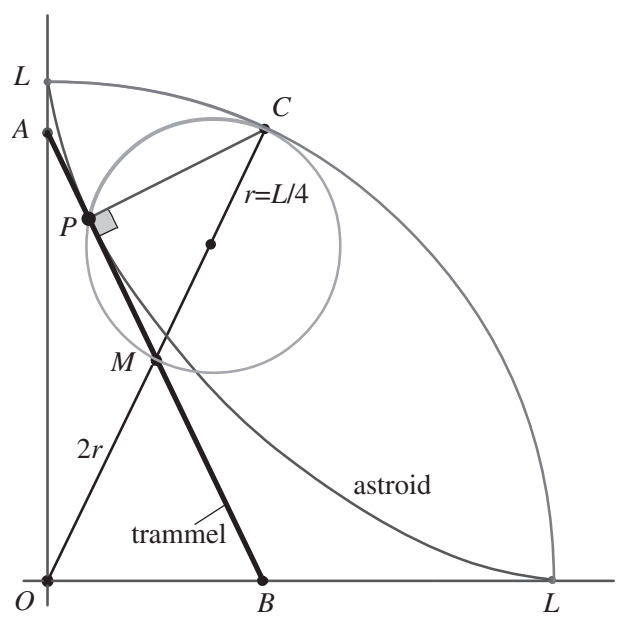

(a)

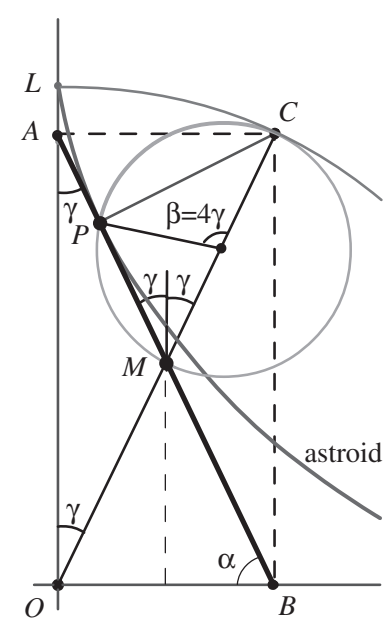

(b)

Figure 4. (a) Point $P$ on the circle of radius $L / 4$ traces an astroid as it rolls inside a circle of radius $L$. (b) Proof that $A B$ has constant length equal to radius $L$.

This property is illustrated in Figure 7a, which shows various positions of the trammel as it slides along the coordinate axes in all four quadrants. It is described by saying that:

The envelope of a moving trammel of fixed length is an astroid.

For calculus derivations of this envelope see [5] and [6].

4. EQUATIONS FOR ELLIPSE, TRAMMEL, AND ASTROID. Figure 5 shows an ellipse with semiaxes $a$ and $b$ and two concentric circles with radii $a$ and $b$. A line 
from the origin making angle $\alpha$ with the $x$ axis is related to the coordinates of a point $E=(x, y)$ on the ellipse by the parametric equations

$$
x=a \cos \alpha, \quad y=b \sin \alpha .
$$

Angle $\alpha$ in (3) is called the eccentric angle of the ellipse (see [1, p. 522]).

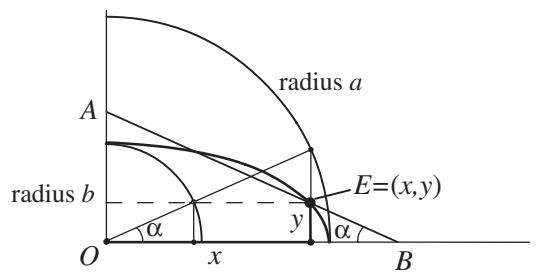

Figure 5. Angle of inclination of a trammel is also the eccentric angle of traced ellipse.

Figure 5 also shows a line segment $A B$ through $E$ making the same angle $\alpha$ with the $x$ axis. It is easy to show that $A B$ has length $a+b$. Therefore it is a trammel whose angle of inclination is the eccentric angle. We simply note that $A E \cos \alpha=x$, so according to (3), $A E=a$. Similarly, $B E \sin \alpha=y$, giving $B E=b$.

In Figure 6a, a line $A B$ of length $L$ makes an angle $\alpha$ with the $x$ axis and is tangent to the astroid at point $P$ with coordinates $(x, y)$. First we note that every point on the trammel satisfies the linear Cartesian equation

$$
\frac{x}{L \cos \alpha}+\frac{y}{L \sin \alpha}=1
$$

because $L \cos \alpha$ and $L \sin \alpha$ are the $x$ and $y$ intercepts of the trammel.

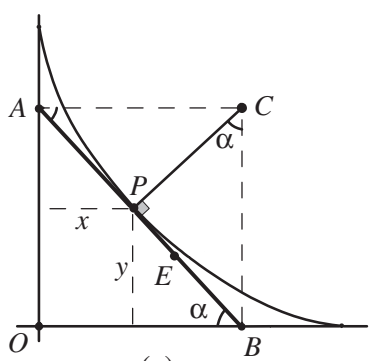

(a)

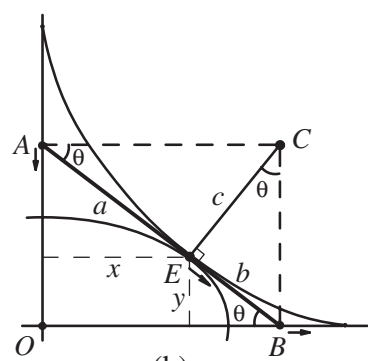

(b)

Figure 6. (a) Finding equations for an astroid. (b) Trammel is simultaneously tangent to the ellipse and the astroid at $E$ when inclined at a special angle $\theta$ given by (8).

To find a Cartesian equation for the astroid we first determine parametric equations expressing the coordinates $(x, y)$ of each point on the astroid in terms of $\alpha$ and $L$. From Figure 6a, we see that

$$
x=A P \cos \alpha, \quad y=B P \sin \alpha .
$$

To express $A P$ and $B P$ in terms of $L$ and $\alpha$, we recall from Figure $4 \mathrm{~b}$ that point $C$ of the rolling circle is a vertex of rectangle $O A C B$. From right triangle $A O B$ in Figure $6 \mathrm{a}$ 
we see that $A C=O B=L \cos \alpha$. From right triangle $A P C$ (inherited from Figure 4) we have $A P=A C \cos \alpha=L \cos ^{2} \alpha$. Similarly we find $P B=L \sin ^{2} \alpha$. Using these in (5) we see that each point $(x, y)$ on the astroid satisfies the parametric equations

$$
x=L \cos ^{3} \alpha, \quad y=L \sin ^{3} \alpha .
$$

Using (6) together with $\cos ^{2} \alpha+\sin ^{2} \alpha=1$ we obtain the following Cartesian equation of the astroid:

$$
\left(\frac{x}{L}\right)^{2 / 3}+\left(\frac{y}{L}\right)^{2 / 3}=1
$$

\section{COMMON TANGENCY OF TRAMMEL, ELLIPSE, AND ASTROID. In} Figure 6a point $P$ with coordinates (5) is on the trammel (4) and on the astroid (7). The trammel is always tangent to the astroid but in general it intersects the ellipse described by (3) at two points as in Figure 5. As the trammel moves, these two points will coincide for some critical angle $\alpha$, say $\alpha=\theta$ as shown in Figure 6b, and in this position the trammel is simultaneously tangent to the ellipse and the astroid at some point $E$ with coordinates $(a \cos \theta, b \sin \theta)$.

The following theorem determines $\theta$ in terms of the semiaxes $a$ and $b$ of the ellipse.

Theorem 1. The trammel is simultaneously tangent to the ellipse and the astroid when its angle of inclination $\theta$ with the $x$ axis satisfies

$$
\tan \theta=\sqrt{\frac{b}{a}} .
$$

Proof. In Figure 6b, a special case of Figure 6a, $C E$ is perpendicular to $A B$, and right triangles $A E C$ and $B E C$ are similar. If $c$ denotes the length of $C E$, then $a / c=c / b$, hence $c^{2}=a b$. Triangle $B E C$ reveals that $\tan \theta=b / c=b / \sqrt{a b}=\sqrt{b / a}$, which proves (8).

Note that point $C$ in Figure $6 \mathrm{~b}$ is also the center of instantaneous rotation of trammel $A B$. As endpoint $A$ moves downward, endpoint $B$ moves to the right, and point $E$ moves in the direction of $A B$ as indicated. This also shows that the trammel is simultaneously tangent to the ellipse and to the astroid at $E$. As $a$ and $b$ vary, keeping $a+b$ constant, the corresponding ellipses are tangent to the astroid. This property, illustrated in Figure 7b, is described as follows:

The envelope of a family of ellipses with $a+b$ constant is an astroid.

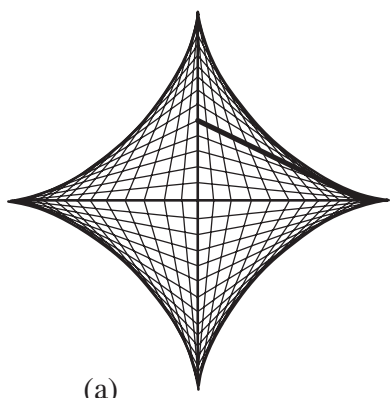

(a)

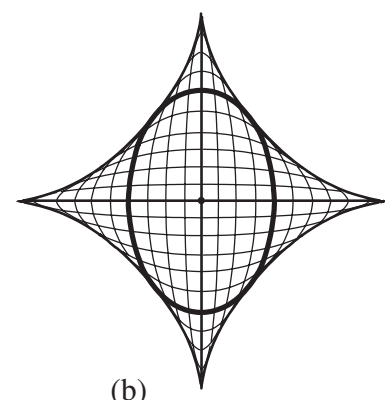

(b)

Figure 7. Astroid as the common envelope of (a) a moving trammel, and (b) a family of traced ellipses. 
A generalization of Theorem 1 to flexible trammels is given in Section 10, leading to a common envelope for a flexible trammel and the curves traced by its points.

6. AREA OF AN ELLIPTICAL SECTOR. The trammel is shown in Figure 8 as a segment $A B$ of length $a+b$ in the first quadrant inclined at an angle $\alpha$ with the $x$ axis. The ellipse is traced by point $E$, where $A E$ has length $a$ and $E B$ has length $b$. As noted earlier, $\alpha$ is the eccentric angle of the ellipse. Let $S(\alpha)$ denote the area of the shaded elliptical sector $O E_{0} E$. Here, $E_{0}$ denotes the position of $E$ when the trammel is horizontal. Then we have the following simple result which we shall deduce without calculus:

(a)

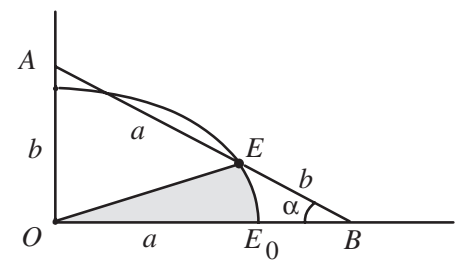

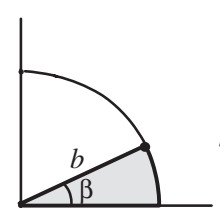

(b)

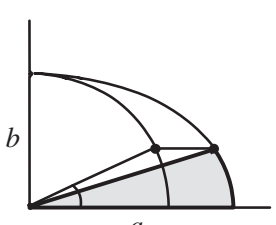

(c)

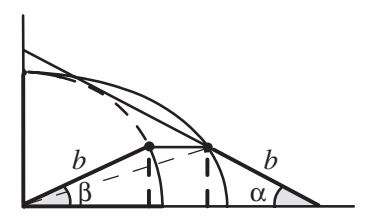

(d)

Figure 8. (a) Elliptical sector $O E_{0} E$ determined by trammel inclined at angle $\alpha$. (b)-(d) Proof that the area of this sector is $a b \alpha / 2$.

Theorem 2. The elliptical sector $O E_{0} E$ has area

$$
S(\alpha)=\frac{1}{2} a b \alpha
$$

Proof. The elliptical sector can be obtained from a circular sector with central angle $\beta$ and radius $b$ (Figure 8b) by horizontal dilation by the factor $a / b$ (Figure $8 \mathrm{c}$ ). The circular sector has area $b^{2} \beta / 2$, so the dilated sector has area $a / b$ times as much or $a b \beta / 2$. But Figure $8 \mathrm{~d}$ shows that $\beta=\alpha$ because both are base angles of congruent right triangles having hypotenuses of equal length $b$ and equal altitudes. Therefore, this simple geometric argument gives (9).

When $a=b$ the ellipse is a circle of radius $b$ and (9) gives the area of a circular sector in terms of the eccentric angle, which now equals the central angle.

The right member of (9) is linear in $\alpha$, so the area of the sector of the ellipse between any two values of $\alpha$, say $0<\alpha_{1}<\alpha_{2} \leq 2 \pi$, is $a b\left(\alpha_{2}-\alpha_{1}\right) / 2$. Thus the area of a more general elliptical sector, such as that shown in Figure 9a, is given by:

$$
\text { Area of general elliptical sector }=\frac{1}{2} a b \varphi,
$$

where $\varphi=\alpha_{2}-\alpha_{1}$ denotes the angle between the two trammel positions, which is also the change in eccentric angle. This charming result describing the area of a general elliptic sector deserves to be better known. In particular, if $\varphi$ is a right angle (two 
perpendicular trammel positions) the area of the sector is $\pi a b / 4$, one quarter of the area of a full ellipse. An example is shown in Figure 9b.

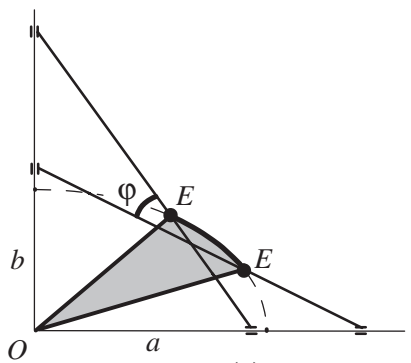

(a)

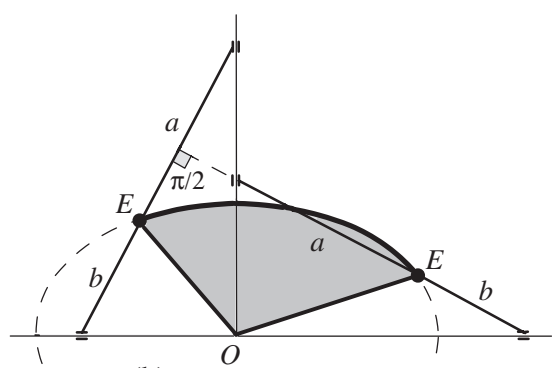

(b)

Figure 9. (a) Area of a general elliptical sector. (b) Special case with $\varphi=\pi / 2$.

7. AREA OF AN ASTROIDAL SECTOR. It is known (see [2, p. 27]) that the area of the region enclosed by the astroid in Figure 10a is 6 times the area of the smaller circular disk that traces the astroid, so in each quadrant the astroidal area is $3 / 2$ times that of the rolling disk.

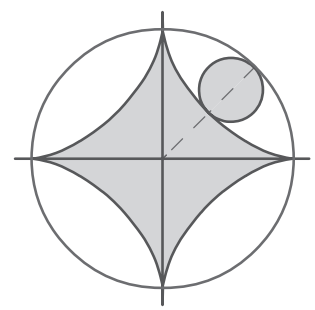

(a)

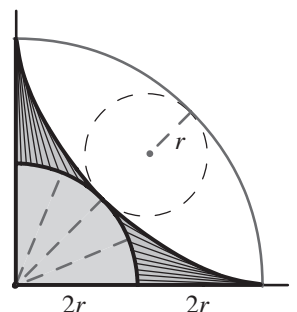

(b)

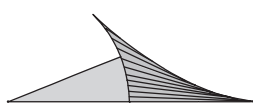

(c)

Figure 10. (a) The region enclosed by an astroid has area 6 times that of the rolling circle. (b) The region in the first quadrant below the astroid has area $3 \pi r^{2} / 2$. (c) Area of an astroidal sector is equal to that of a circular sector.

Figure 10b shows the region in the first quadrant composed of a quarter of a disk of radius $2 r$ and area $\pi r^{2}$, together with two congruent shaded regions between the disk and the astroid. The rolling disk has radius $r$. We will show that each shaded region has area $\pi r^{2} / 4$, so the two regions together with the quarter disk have area $3 \pi r^{2} / 2$. The surprising equality of the area of an astroidal sector with that of a circular sector is illustrated in Figure 10c. Before verifying the formulas, we mention an application to sliding doors.

Comparing a hinged door with a sliding door. From Figure $10 \mathrm{~b}$ we see that a door of length $4 r$ hinged at the origin sweeps out floor space of area $4 \pi r^{2}$. By comparison, a door of the same length sliding along perpendicular tracks sweeps out floor space of area $3 \pi r^{2} / 2$, which is $3 / 8$ of $4 \pi r^{2}$, an impressive saving of $62.5 \%$. Examples involving sliding doors that fold are discussed in Section 8.

The area of an astroidal sector will be deduced from a more general result, illustrated in Figure 11a, where the shaded region is swept by the portion of the trammel 
cut off by the quarter disk of radius $2 r$ as the trammel moves from a horizontal position to a general angle of inclination $\alpha$ as shown. We call this region a general astroidal sector and denote its area by $A_{r}(\alpha)$. This region is an example of a tangent sweep, and Figure $11 \mathrm{~b}$ shows the corresponding tangent cluster, in which the tangent trammel segments have been translated so that each point of tangency is moved to a common point $Q$. Mamikon's sweeping tangent theorem ([3, p. 900]) tells us that the tangent sweep and the tangent cluster have equal areas. Using Figure 11 we shall prove:

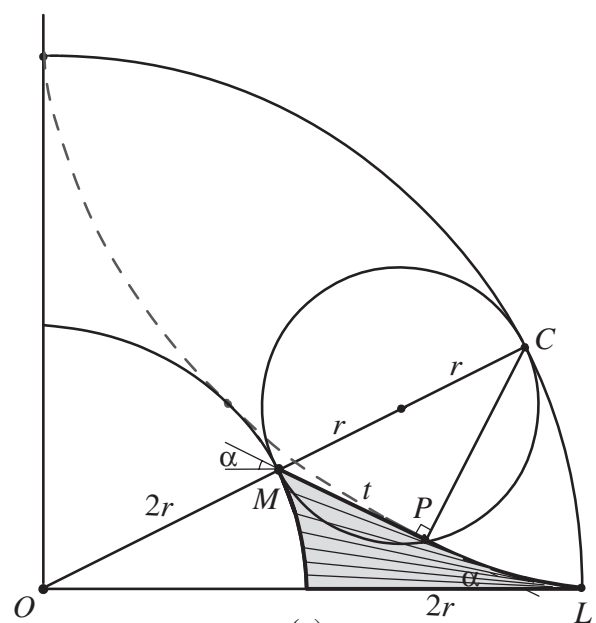

(a) (d)

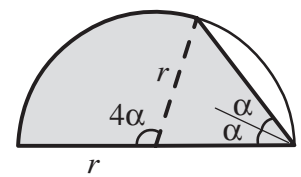

(c)

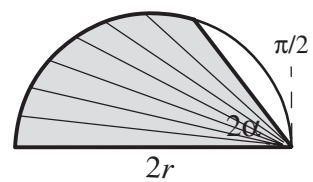

$\pi / 4$

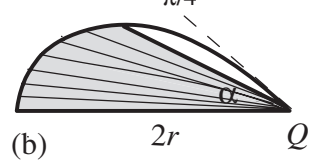

Figure 11. Calculating the area of a general astroidal sector.

Theorem 3. The area of the general astroidal sector is given by

$$
A_{r}(\alpha)=r^{2} \alpha+\frac{1}{4} r^{2} \sin 4 \alpha .
$$

Proof. Figure 11a shows the larger circle of radius $L$ centered at the origin, and the smaller circle of radius $r=L / 4$ rolling inside it, with $P$ denoting the point on the astroid when the trammel has turned through angle $\alpha$ from the horizontal. Point $M$, the midpoint of $O C$, is also on the smaller circle as shown, and $t$ denotes the length of the tangent segment $P M$. The argument used in Figure 4a shows that angle $P M C$ is equal to $2 \alpha$, so length $t=2 r \cos 2 \alpha$. Therefore the tangent cluster shown in Figure $11 \mathrm{~b}$ is bounded by a portion of a rosette whose polar coordinates $(t, \alpha)$ relative to the origin at $Q$ satisfy $t=2 r \cos 2 \alpha$.

The area of the rosette portion in (b) can be easily calculated using integral calculus, giving (10). It can also be determined without integration by noting that this area is half that of the portion of a semicircular disk enclosed by the inscribed angle $2 \alpha$ in (c). To see why, note that each tiny wedge with vertex $Q$ in (b) also appears in (c) with its vertex angle doubled. The region in (c), also shown in (d), is the union of the circular sector subtended by a central angle $4 \alpha$, with area $2 r^{2} \alpha$, and an isosceles triangle with legs $r$ and vertex angle $2 \alpha$, and with area $\frac{1}{2} r^{2} \sin 4 \alpha$. Thus the area of the region in (b), hence that of the general astroidal sector in (a), is given by (10). When $\alpha=\pi / 4$ this gives $\pi r^{2} / 4$ for each astroidal sector in Figure 10b, and proves the surprising area equality in Figure 10c.

Application: Area of the region swept by any portion of a trammel. Figure 12a shows a trammel of length $a+b$ simultaneously tangent to an ellipse with semiaxes 

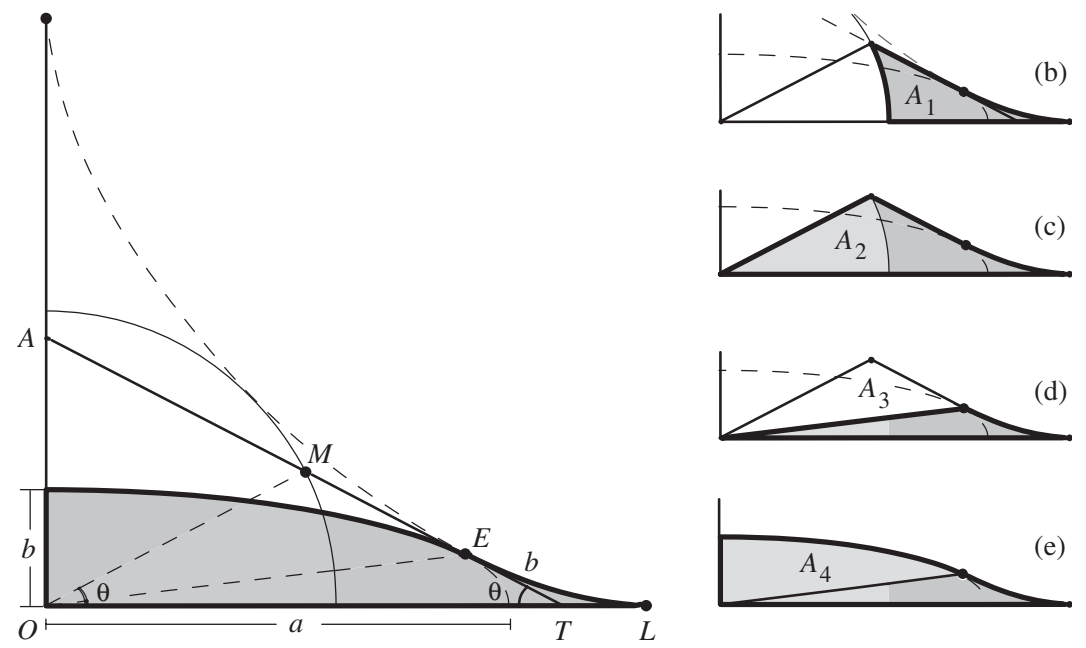

(a)

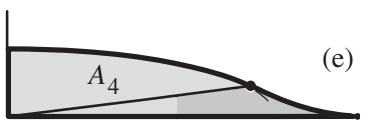

Figure 12. Region (a) swept by a portion of a trammel of length $b$ constructed in (b)-(e).

$a$ and $b$ and to an astroid at point $E$ where it makes a critical angle $\theta$ with the $x$ axis as determined by (8). As applications of (9) and (10) we shall calculate, in terms of $a$, $b$, and $\theta$, the area $A(\theta)$ of the region swept out by the trammel segment of length $b$ as the trammel slides from horizontal to vertical position. The region in question, under the ellipse and the astroid, is shown shaded in Figure 12a. We build this region in four steps as shown in Figures 12b-e.

The shaded region in Figure $12 \mathrm{~b}$, whose area we denote by $A_{1}$, is like the astroidal sector in Figure 11a cut off by the circle of radius $2 r=(a+b) / 2$ with $\alpha=\theta$. Figure $12 \mathrm{c}$ shows the astroidal sector of Figure $12 \mathrm{~b}$ adjacent to a circular sector whose area we denote by $A_{2}$. In Figure $12 \mathrm{~d}$ a triangle of area $A_{3}$ has been removed from the region of Figure 12c. In Figure 12e an elliptical sector of area $A_{4}$ is added to the region of Figure 12d, thus completing the shaded region in Figure 12a. Hence

$$
A(\theta)=A_{1}+A_{2}-A_{3}+A_{4},
$$

and now we calculate each area $A_{i}$ separately.

From (10) we find $A_{1}=A_{r}(\theta)=r^{2} \theta+\frac{1}{4} r^{2} \sin 4 \theta$. Point $M$ is the midpoint of the trammel, so $O M$ also makes an angle $\theta$ with the $x$ axis, and hence the circular sector in Figure $12 \mathrm{c}$ has area $A_{2}=2 r^{2} \theta$. In Figure $12 \mathrm{~d}$ the area of the triangle is the difference of areas of triangles $O M T$ and OET in Figure 12a. They have a common base $(a+b) \cos \theta$ and respective altitudes $2 r \sin \theta$ and $b \sin \theta$. Hence we see that area $A_{3}=(a+b)\left(r-\frac{b}{2}\right) \sin \theta \cos \theta=r(2 r-b) \sin 2 \theta$. Finally, from (9) we infer that the elliptical sector in Figure $12 \mathrm{e}$ has area $A_{4}=\pi a b / 4-a b \theta / 2$. Using these values in (11) we find area $A(\theta)$ as the following Corollary of Theorems 1, 2, and 3:

Corollary 1. If $r=(a+b) / 4$, and $\theta$ is given by (8), then

$$
A(\theta)=3 r^{2} \theta+\frac{r^{2}}{4} \sin 4 \theta-r(2 r-b) \sin 2 \theta+\frac{a b}{2}\left(\frac{\pi}{2}-\theta\right) .
$$

8. ZIGZAG TRAMMEL. Start with a standard trammel $Z T$ of length $L$ regarded as a rod initially on the positive $x$ axis, with $Z$ at $(0,0)$ and $T$ at $(L, 0)$, as shown in Figure 13a. 


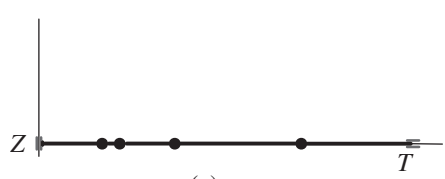

(a)

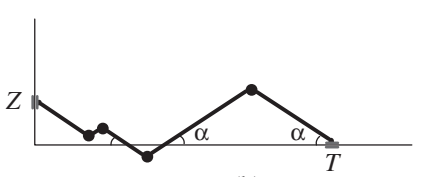

(b)

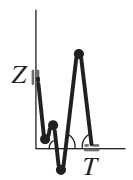

(c)

Figure 13. (a) Standard trammel of length $L$ divided into rods. (b) and (c) Zigzag trammel of length $L$, with all rods making equal angles with the $x$ axis.

Divide $Z T$ into a finite number of hinged rods of arbitrary lengths whose sum is $L$. Now let the free ends $T$ and $Z$ slide along the respective coordinate axes as indicated in Figures 13b, c, with all rods making equal angles with the $x$ axis. We call this a zigzag trammel if the number of rods is greater than 1. In the examples shown, angle $\alpha$ is allowed to increase from 0 to $\pi / 2$, so the rightmost rod will stay in the first quadrant.

For any fixed $\alpha>0$, an ant starting at $T$ and walking along the zigzag trammel toward $Z$ decreases its $x$ coordinate monotonically, but its $y$ coordinate is piecewise monotonic, alternately increasing or decreasing as it crosses the hinges.

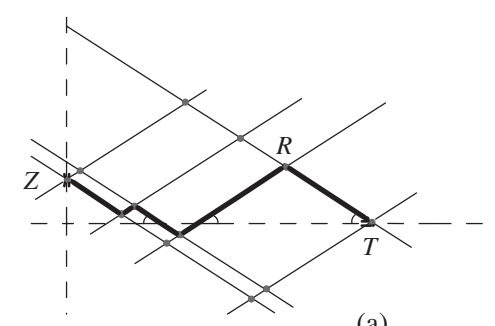

(a)

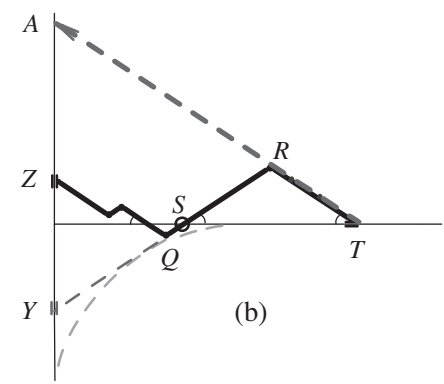

Figure 14. (a) Parallel zig lines and parallel zag lines through the hinges of a zigzag trammel. (b) A line through the rightmost $\operatorname{rod} R T$ intersects the $y$ axis at $A$.

Now refer to Figure 14. Any line parallel to the rightmost rod $R T$ is called a zig line, while those parallel to the rod adjacent to $R T$ are called zag lines. Because all rods make equal angles with the $x$ axis, they generate a finite set of parallel zig lines and another finite set of parallel zag lines. Each hinge is the intersection of a zig line and a zag line, as indicated in Figure 14a. The configuration is like a movable latticework in which any two adjacent rods determine a parallelogram.

In Figure 14b, $A$ denotes the point at which a line through the rightmost $\operatorname{rod} R T$ intersects the $y$ axis. By parallel projection suggested by Figure 14a, it follows that the length of $A T$ is the sum of the lengths of the rods of the zigzag trammel. Consequently, we have the following property:

Property 1. As a zigzag trammel ZT of length $L$ slides along the axes, a line segment $A T$ through the rightmost rod $R T$, from the $y$ axis to $T$, is a standard trammel of length $L$ sliding along the same axes, regardless of the number of rods and their relative sizes.

Applications to folding doors. Zigzag trammels can be realized physically as folding doors. Examples are those treated in [5], where the left endpoint $Z$ is kept fixed. The seemingly unexpected appearances of the same astroid and various ellipses associated with bifold closet doors treated in [5] are easily explained as consequences of Property 1. In [5] the floor area swept out by the right panel of a bifold closet door 
was found using calculus. That result follows directly from the discussion related to Figures 10b and 10c. It is also a special case of (12) when $b=a=L / 2$ and $\theta=\pi / 4$. As we observed earlier, using a one-panel door sliding along two perpendicular tracks instead of a hinged door results in a saving of $62.5 \%$ in swept floor area. Using a bifold closet door instead of a one-panel sliding door saves only an additional $6.25 \%$.

If the two rods joining alternate hinges of a zigzag trammel always have equal lengths, they form an isosceles triangle whose vertex is at the intermediate hinge. This configuration occurs when closet doors are divided into two or more hinged panels that fold or unfold as the door is opened or closed. Equation (12) can be used in particular to calculate the floor area swept by the rightmost panel of a door of $n$ equal-sized panels by taking $b=L / n$ and $a=L(n-1) / n$. We omit the details.

A zigzag trammel has other interesting properties. For example, consider the rod $R Q$ in Figure $14 \mathrm{~b}$ adjacent to the rightmost $\operatorname{rod} R T$. Let $S$ denote the point where $R Q$ or its extension to point $Y$ on the $y$ axis intersects the $x$ axis. Then we have:

Property 2. As a zigzag trammel ZT of length $L$ slides along the axes, the point $S$ remains on the $x$ axis and $S Y$ has constant length $L-2 R T$, so segment $S Y$ is a standard trammel sliding along the same axes.

Proof. During the entire motion, triangle $T R S$ is isosceles, with $R S=R T$, so $S$ remains on the $x$ axis. Also $S Y=Y R-S R=(L-R T)-S R=L-2 R T$.

The same analysis applies to each of the rods of a zigzag trammel. A line through the rod extended to meet the $y$ axis produces a standard "mini-trammel" whose ends slide along the same axes. Consequently, each point on such a mini-trammel traces its own ellipse. It also has its own astroid as an envelope. One of them is shown by the dashed curve in Figure 14b. The next theorem tells how to determine the length of each such mini-trammel.

Theorem 4. Consider a zigzag trammel of length $L$ with rods of lengths $z_{1}, z_{2}, \ldots, z_{n}$, labeled from right to left. Then the segment between the $x$ and $y$ axes of the line through the ith rod is a standard trammel whose length $L_{i}$ is given as follows:

for odd $i$ (zig lines),

$$
L_{1}=L, \quad L_{2 k+1}=L-2\left(z_{2}+z_{4}+\cdots+z_{2 k}\right), \quad k \geq 1,
$$

and for even $i$ (zag lines),

$$
L_{2 k}=L-2\left(z_{1}+z_{3}+\cdots+z_{2 k-1}\right), \quad k \geq 1 .
$$

An inductive proof can be given, which is omitted. The formulas for $L_{1}$ and $L_{2}$ to begin the induction are contained in Properties 1 and 2, respectively.

A knowledge of the length of a mini-trammel determines its corresponding astroidal envelope, and also determines the semiaxes of the ellipse traced by any point on the line through each rod. The semiaxes are the distances from the tracing point to the endpoints of the corresponding mini-trammel. In particular the semiaxes $a_{k}$ and $b_{k}$ of the ellipse traced by the hinge joining rod $k$ with rod $k+1$ are given by

$$
a_{k}=L-\left(z_{1}+\cdots+z_{k}\right), \quad b_{k}=\left|L_{k}-a_{k}\right|,
$$

where $L_{k}$ is the trammel length determined by Theorem 4 .

For animations showing zigzag trammels in motion see [4]. 
9. FLEXIBLE TRAMMEL. An astroid is the common envelope of a moving standard trammel and a family of traced ellipses (Figure 7). Now we introduce trammels of variable length that provide a new solution of a classical problem of finding the envelope of a family of straight lines. Theorem 7 reveals that this is also the envelope of a family of curves traced by special points on these lines. Sections 11 and 12 provide many interesting examples.

Figure 15a shows the first quadrant segment of a line with Cartesian equation

$$
\frac{x}{m}+\frac{y}{n}=1 .
$$

Here $m$ and $n$ are the $x$ and $y$ intercepts of the line. When both intercepts are nonzero they can be regarded as legs of a right triangle of lengths $|m|$ and $|n|$ having hypotenuse of length $\sqrt{m^{2}+n^{2}}$. If $m^{2}+n^{2}$ is constant, the line segment joining the axes will have constant length and we obtain a standard trammel of that length.

Instead of $m^{2}+n^{2}$ being constant, we assume that the intercepts $(m, n)$ satisfy a more general relation, say

$$
G(x, y)=0,
$$

where $G$ is a prescribed function we call the governor of the trammel. If the intercepts $m$ and $n$ satisfy $G(m, n)=0$, we refer to the portion of line (13) in a given quadrant as a flexible trammel governed by $G$. The length of the trammel is equal to that of the radial segment from the origin to $(m, n)$, where $G(m, n)=0$. This segment and the trammel are the diagonals of a rectangle with opposite vertices at the origin and at $(m, n)$ as shown in Figure 15b. This extends Figure 2c for a standard trammel.

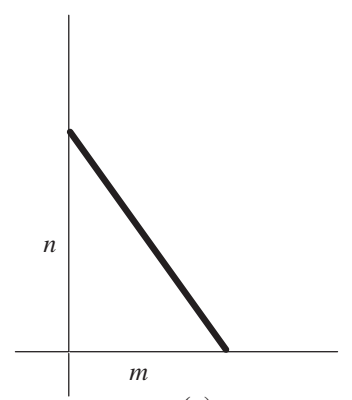

(a)

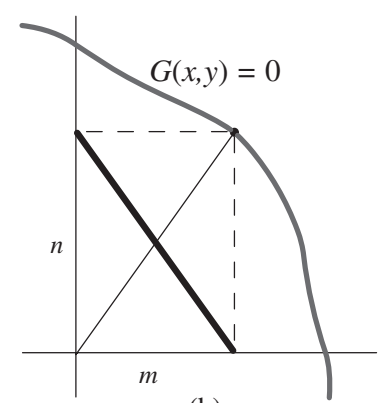

(b)

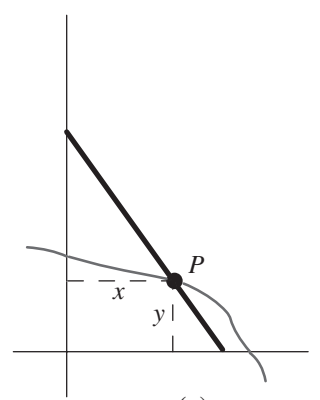

(c)

Figure 15. (a) Trammel with intercepts $m$ and $n$. (b) Point $(m, n)$ is on the governing curve $G(x, y)=0$. (c) A point $P$ that divides the trammel into segments of prescribed ratio $\lambda / \mu$ with $\lambda+\mu=1$ lies on the path given by $G(x / \lambda, y / \mu)=0$.

When $G(m, n)=m^{2}+n^{2}-1$, the trammel has constant length 1 (it is a standard trammel) and any point $P$ on it traces an ellipse. Now we seek the path traced by any point $P$ on a flexible trammel as the trammel slides along the axes. We treat only the simple case when $P$ divides the trammel into two pieces whose lengths are in constant ratio. It can be realized physically by an elastic string with a knot on it.

Theorem 5. Consider a flexible trammel governed by a function $G$. A point $P$ that divides the trammel into two pieces whose lengths are in a prescribed ratio $\lambda / \mu$, where $\lambda$ and $\mu$ are nonzero constants with $\lambda+\mu=1$, lies on the path given by

$$
G\left(\frac{x}{\lambda}, \frac{y}{\mu}\right)=0
$$


Proof. Point $P$ has coordinates $(\lambda m, \mu n)$, and because of (14) it lies on the path given by (15).

Definition. The graph of (15) is called the trace of $P$ and we denote it by $\tau(\lambda)$.

Theorem 5 shows how easy it is to determine the trace of $P$ once the governing function of the trammel is known.

Because $\lambda+\mu=1$, the curves $\tau(\lambda)$ form a one-parameter family of curves determined by the governor $G$ and the choice of $\lambda$. Trace $\tau(\lambda)$ is obtained by dilating the graph of $G$ horizontally by a factor $\lambda$ and vertically by a factor $\mu=1-\lambda$.

If both $m$ and $n$ are positive, the trammel lies in the first quadrant. The restriction $\lambda+\mu=1$ keeps point $P=(\lambda m, \mu n)$ in the first quadrant between the ends of the trammel if both $\lambda$ and $\mu$ are positive. But if $\lambda>1$ then $\mu=1-\lambda$ is negative and $P$ lies in the fourth quadrant. Similarly, if $\mu>1$ then $\lambda=1-\mu$ is negative and $P$ lies in the second quadrant.

10. TANGENCY OF A FLEXIBLE TRAMMEL AND ITS TRACE. In Section 5 we found the point of tangency of a standard trammel and the ellipse traced by a given point on the trammel. Now we do the same for the curve in (15) traced by a point $P$ that divides a flexible trammel into pieces of prescribed ratio $\lambda / \mu$. For simplicity, assume that the governing equation $G(x, y)=0$ in (14) can be solved for $y$ in terms of $x$, giving

$$
y=g(x)
$$

Then the equation of the trace of any point $P=(\lambda m, \mu n)$ on the trammel is

$$
y=\mu g\left(\frac{x}{\lambda}\right) .
$$

The curve in (17) has slope

$$
\frac{d y}{d x}=\frac{\mu}{\lambda} g^{\prime}\left(\frac{x}{\lambda}\right)
$$

at each of its points, while the trammel has slope $-n / m$ at each of its points. Equating the slopes at the point $P$ of intersection we find

$$
-\frac{n}{m}=\frac{\mu}{\lambda} \frac{d n}{d m}
$$

where $d n / d m=g^{\prime}(m)$.

Equation (18) determines the ratio $n / m$ implicitly in terms of $\mu, \lambda$, and $G$, and we denote by $\rho$ the ratio $n / m$ so defined. The ratio $\rho$ is the tangent of the critical angle $\theta$ that the trammel makes with the $x$ axis when it is tangent to the trace $\tau(\lambda)$. Consequently, (18) gives the following extension of Theorem 1:

Theorem 6. Wherever the trammel is tangent to the trace its angle of inclination $\theta$ with the $x$ axis satisfies

$$
\tan \theta=\rho
$$

where $\rho$ depends on $\mu, \lambda$, and $G$. 
To show how (19) can be used to determine the slope of the trammel when it is tangent to the trace $\tau(\lambda)$ we consider the following example.

Example 1. $G(x, y)=\left(\frac{x}{A}\right)^{k}+\left(\frac{y}{B}\right)^{k}-1$ ( $k$ th power ellipse).

The trace in this example is given by

$$
\left(\frac{x}{\lambda A}\right)^{k}+\left(\frac{y}{\mu B}\right)^{k}=1 .
$$

The equation $G(m, n)=0$ becomes $m^{k} B^{k}+n^{k} A^{k}=A^{k} B^{k}$, which yields

$$
\frac{d n}{d m}=-\frac{B^{k} m^{k-1}}{A^{k} n^{k-1}} .
$$

Equation (18) implies $(n / m)^{k}=(B / A)^{k}(\mu / \lambda)$, and when this is solved for $\rho=n / m$ we obtain

$$
\tan \theta=\rho=\frac{B}{A}\left(\frac{\mu}{\lambda}\right)^{1 / k} .
$$

When $k=2$ and $A=B$ this agrees with the formula obtained earlier in (8).

The case $k=1$ is also of interest because the governor is a straight line $x / A+$ $y / B=1$ and every trace is also a straight line $x /(\lambda A)+y /(\mu B)=1$. In this case (21) becomes

$$
\tan \theta=\frac{n}{m}=\frac{B}{A}\left(\frac{\mu}{\lambda}\right)
$$

The trammel and trace are straight lines that intersect at exactly one point unless they have the same slope, in which case the trammel and trace coincide. From (22) we see that this happens if and only if $\mu / \lambda=A n / B m$.

11. ENVELOPE OF A TRAMMEL AND OF ITS FAMILY OF TRACES. As the angle $\alpha$ of inclination of a trammel varies, it generates a one-parameter family of lines that has an envelope $\varepsilon$ touching all of them. For a given position of the trammel, select the point of tangency with $\varepsilon$. That point divides the trammel into pieces having a certain ratio $\lambda / \mu$ with $\lambda+\mu=1$. Point $P=(\lambda m, \mu n)$ traces a curve $\tau(\lambda)$ given by (17). This trace cannot cross $\varepsilon$ in a neighborhood of $P$, otherwise the trammel would cross its envelope at $P$. Therefore that trace is also tangent to $\varepsilon$ at $P$. If envelope $\varepsilon$ exists we can use (18) and (13) to determine parametric equations for it. First, we have

$$
\frac{x}{m}=\lambda=\frac{\lambda}{\lambda+\mu}=\frac{1}{1+\frac{\mu}{\lambda}},
$$

which in view of (18) and (13) gives us the pair of equations

$$
\frac{x}{m}=\frac{1}{1-\frac{n}{m} \frac{d m}{d n}}, \quad \frac{y}{n}=1-\frac{x}{m} .
$$

This gives us the following: 
Theorem 7. The envelope of a moving flexible trammel is also the envelope of the family of traces of the trammel, as described by (23).

The second equation in (23) can also be written as

$$
\frac{y}{n}=\frac{1}{1-\frac{m}{n} \frac{d n}{d m}} .
$$

Because $m$ and $n$ satisfy the governing condition $G(m, n)=0$, relations (23) and (24) serve as parametric equations for the envelope $\varepsilon$ in terms of $m$ or $n$.

In general the derivatives $d m / d n$ and $d n / d m$ can be obtained by differentiating the implicit equation $G(m, n)=0$, as is done for the following examples.

Example 1. $G(x, y)=\left(\frac{x}{A}\right)^{k}+\left(\frac{y}{B}\right)^{k}-1$ (kth power ellipse).

In this case we use (20) for $d n / d m$, and the parametric equations in (23) and (24) yield $x / A=(m / A)^{k+1}, y / B=(n / B)^{k+1}$. Eliminating the parameters $m, n$ we find that the envelope of the family of $k$ th power ellipses is a generalized astroid given by

$$
\left(\frac{x}{A}\right)^{k /(k+1)}+\left(\frac{y}{B}\right)^{k /(k+1)}=1 .
$$

Figure 16 shows some special cases. The dashed line in Figure 16a shows the case $k=1$ with $A=2, B=3$. In this case the envelope $\varepsilon$ is the parabola described by

$$
\sqrt{x / 2}+\sqrt{y / 3}=1
$$

The symmetric case $A=B$ (Figure 16d) yields a known parabolic envelope [6, p. 76]. Figure $16 \mathrm{~b}$ shows the case $k=2$ (an ordinary ellipse with $A=2, B=3$ ) whose envelope is an asymmetric astroid.

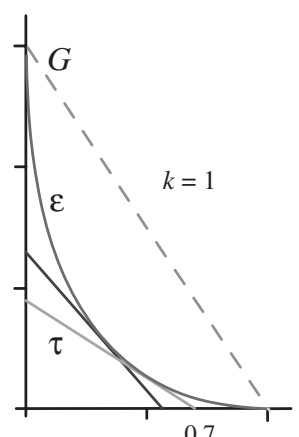

(a)

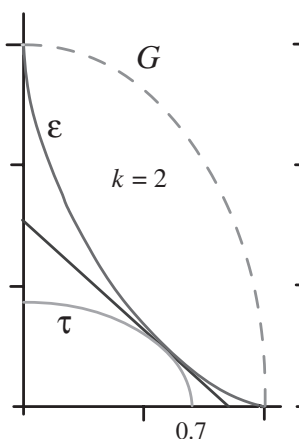

(b)

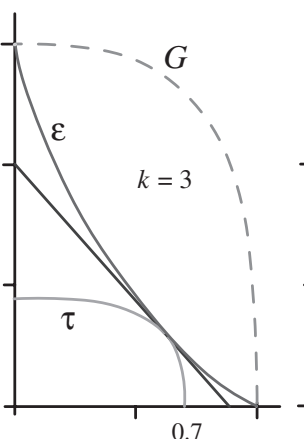

(c)

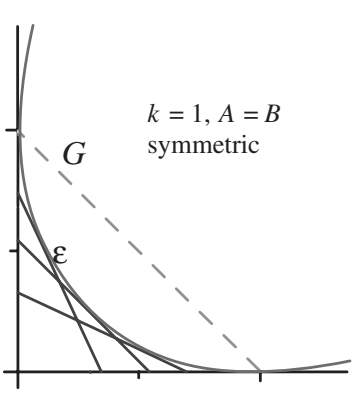

(d)

Figure 16. Special cases of Example 1 showing the governor $G$ (dashed) and the trammel tangent to the envelope $\varepsilon$. In (a)-(c), the trace curve $\tau(0.7)$ is shown. In (d) the governor is $x+y=1$, shown in the first quadrant. When the governor is in the second or fourth quadrant, the parabolic envelope continues in the first quadrant with the line $y=x$ as its axis of symmetry. 
Example 2. $G(x, y)=y-x^{k}$ ( $k$ th power function).

In this case the trace is another $k$ th power function given by

$$
y=\mu\left(\frac{x}{\lambda}\right)^{k} .
$$

Figure 17a shows the case $k=1$, in which the governor is the line $y=x$, the trace curve is the line $y=\mu x / \lambda$, which is never tangent to the trammel, and there is no envelope. If $k \neq 1$, the envelope is yet another $k$ th power function given by

$$
y=\frac{\left(\frac{k-1}{k}\right)^{k}}{1-k} x^{k} .
$$

Moreover, in this case the envelope touches all the trace curves only at the origin. The line through the trammel is always tangent to the envelope in the fourth quadrant.

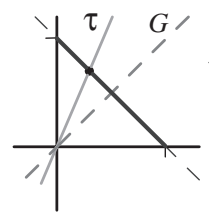

(a) $k=1$

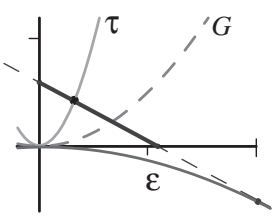

(b) $k=2$

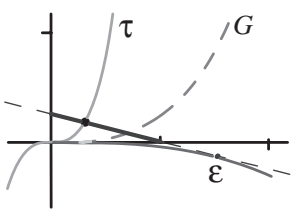

(c) $k=3$

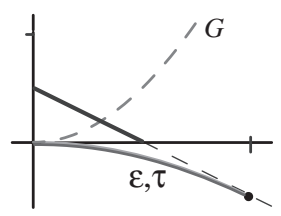

(d)

Figure 17. Special cases of Example 2 with positive $k$, and $\lambda=0.3$ in (a)-(c).

Figure $17 \mathrm{~d}$ shows a special feature that holds for any exponent $k$. The trammel and the trace have the same slope at their point of intersection when (19) is satisfied. If $n=m^{k}$ then $d n / d m=k m^{k-1}=k n / m$, and (19) implies

$$
\frac{n}{m}\left(\frac{\mu k}{\lambda}+1\right)=0 \text {. }
$$

Relation (26) is satisfied if $n / m=0$ (when the trammel is horizontal) but (26) also holds for a nonhorizontal trammel when the point of subdivision $(\lambda m, \mu n)$ on the trammel satisfies $\lambda / \mu=-k$, or $\lambda=k /(k-1), k \neq 1$. For this choice of $\lambda$, the single trace $\tau(\lambda)$ coincides with the envelope of the family of traces, and a line through the trammel is tangent to both in the fourth quadrant as shown in Figure 17d.

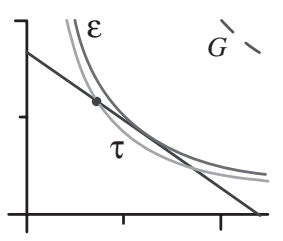

(a) $k=-1$

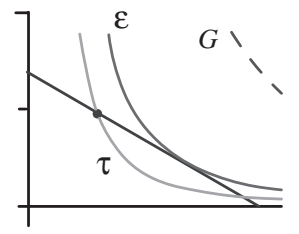

(b) $k=-2$

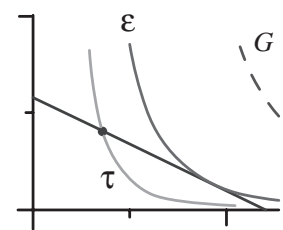

(c) $k=-3$

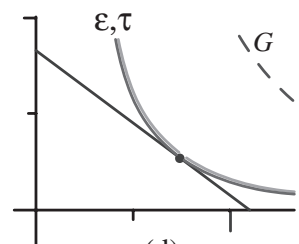

(d)

Figure 18. Special cases of Example 2 with negative $k$, and $\lambda=0.3$ in (a)-(c).

Figure 18 shows special cases of Example 2 when the exponent $k$ is negative. In Figure 18a, b, c we have $k=-1, k=-2, k=-3$, respectively. The exceptional case 
(26) is shown in Figure 18d. For the special choice of ratio $\lambda / \mu=-k$, the trace $\tau(\lambda)$ coincides with the envelope and the trammel is tangent to both in the first quadrant as indicated in Figure 18d.

The governing curve, each trace, and the envelope in Figure 18a are rectangular hyperbolas with the axes as asymptotes.

The next example treats $k$ th power governing hyperbolas written in standard form.

Example 3. $G(x, y)=\left(\frac{x}{A}\right)^{k}-\left(\frac{y}{B}\right)^{k}-1(k$ th power hyperbola, $k \neq 0)$.

By Theorem 5 the trace is also a generalized $k$ th power hyperbola given by

$$
\left(\frac{x}{\lambda A}\right)^{k}-\left(\frac{y}{\mu B}\right)^{k}=1 .
$$

If $k \neq-1$, the envelope has Cartesian equation resembling that in (25) with a difference instead of a sum:

$$
\left(\frac{x}{A}\right)^{k /(k+1)}-\left(\frac{y}{B}\right)^{k /(k+1)}=1 .
$$

\section{APPLICATION: GRAPHIC CONSTRUCTION OF ENVELOPES AND} GOVERNORS AS A CLASSROOM ACTIVITY. The foregoing examples suggest a simple and engaging educational activity that can be performed on graph paper. It reveals the qualitative shape of the envelope of a family of tangent lines without the use of equations for the envelope.

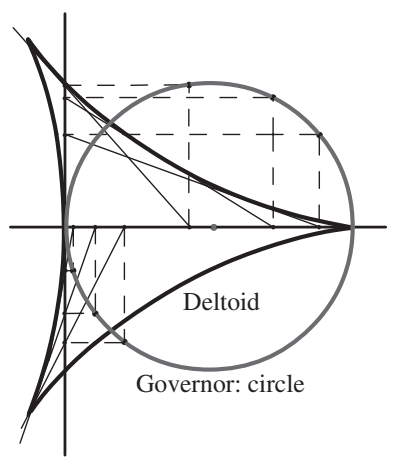

(a) Deltoid as envelope

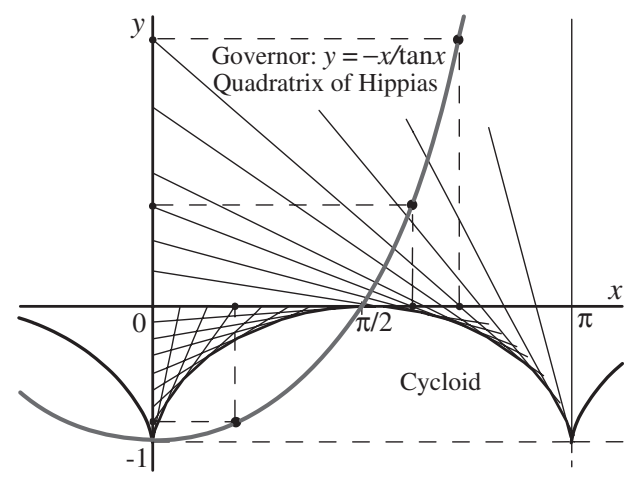

(b) Cycloid as envelope

Figure 19. (a) A circular governor tangent to the $y$ axis produces a deltoid as envelope. (b) Cycloid as envelope for quadratrix of Hippias as governor. Different branches of the quadratrix produce copies of the cycloid.

Start with a straight line as governor $G$, as shown by the dashed line in Figure 16a. Choose any point $(m, n)$ on $G$, and drop perpendiculars to the coordinate axes to obtain the projections $(m, 0)$ and $(0, n)$ on the axes. The line drawn through these projected points has intercepts $m$ and $n$ and is tangent to the corresponding envelope $\varepsilon$. By choosing several points on $G$ and repeating this process, the positions of the tangent lines gradually reveal the shape of the envelope.

For a more general governor $G$ the corresponding envelope $\varepsilon$ can be constructed in exactly the same manner. The following examples are of special interest.

1. If $G$ is a straight line, $\varepsilon$ is a parabola (Figures 16 a and d).

2. If $G$ is a circle centered at the origin (as in Figure 16b), $\varepsilon$ is an astroid. 
3. If $G$ is a circle tangent to the $y$ axis with center on the $x$ axis as shown in Figure $19 \mathrm{a}$, the envelope is a deltoid (see [2]).

4. If $G$ is the quadratrix of Hippias, known since $430 \mathrm{BC}$, (see [6, p. 204]), $\varepsilon$ is a cycloid (Figure 19b). This connection between the quadratrix of Hippias and the cycloid is completely unexpected.

We can also start with a prescribed curve as envelope and determine the governor leading to this envelope by simply reversing the steps described above, as illustrated in Figure 20 for the following examples.

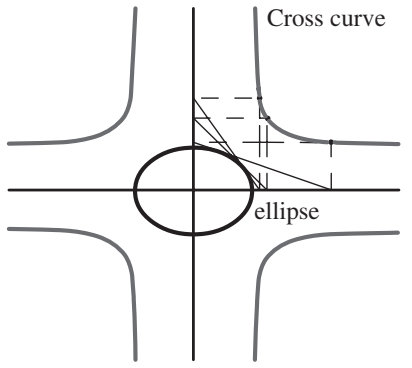

(a) Cross curve as governor

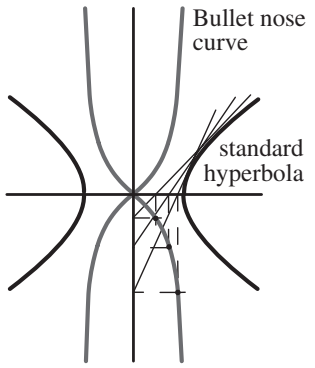

(b) Bullet nose curve as governor

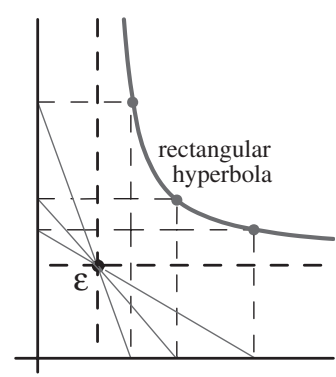

(c) Single point as envelope

Figure 20. (a) An ellipse as envelope arising from a cross curve. (b) A hyperbola as envelope arising from a bullet nose curve. (c) A single point as envelope of a "shifted" hyperbola.

5. When envelope $\varepsilon$ is an ellipse centered at the origin as shown in Figure 20a, the governor is a cross curve (see [6, p. 203]).

6. When envelope $\varepsilon$ is a hyperbola centered at the origin as shown in Figure 20b, the governor is a bullet nose curve (see [6, p. 203]).

7. When envelope $\varepsilon$ is a single point, the governor is a rectangular hyperbola with asymptotes intersecting at that point (Figure 20c).

Also, if the trammel is realized by an elastic string tangent to the envelope, a knot at its midpoint will trace a curve similar to the governor. A knot elsewhere will trace a dilated copy of the governor (Theorem 5).

\section{REFERENCES}

1. T. M. Apostol, Calculus, vol. 1, 2nd ed., John Wiley, New York, 1967.

2. T. M. Apostol and M. A. Mnatsakanian, Generalized cyclogons, Math Horizons September (2002) 25-28.

3. — Tangents and subtangents used to calculate areas, this MONTHLY 109 (2002) 900-908.

4. M. A. Mnatsakanian, Zigzag trammel (2007), available at http://www . mamikon. com/Trammel .html.

5. D. Seiple, E. Boman, and R. Brazier, Mom! There's an astroid in my closet!, Math. Mag. 80 (2007) 104111.

6. R. C. Yates, A Handbook on Curves and Their Properties, J. W. Edwards, Ann Arbor, MI, 1947.

TOM M. APOSTOL joined the Caltech mathematics faculty in 1950 and became professor emeritus in 1992. $\mathrm{He}$ is director of Project MATHEMATICS! (described at the website http://www.projectmathematics.

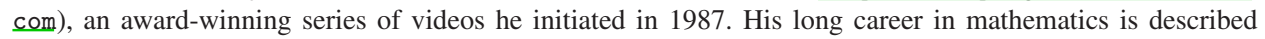
in the September 1997 issue of The College Mathematics Journal. He is currently working with colleague Mamikon Mnatsakanian to produce materials demonstrating Mamikon's innovative and exciting approach to mathematics.

California Institute of Technology, 253-37 Caltech, Pasadena, CA 91125

apostol@caltech.edu 
MAMIKON A. MNATSAKANIAN received a Ph.D. in physics in 1969 from Yerevan University, where he became professor of astrophysics. As an undergraduate he began developing innovative geometric methods for solving many calculus problems by a dynamic and visual approach that makes no use of formulas. He is currently working with Tom Apostol under the auspices of Project MATHEMATICS! to present his methods in a multimedia format.

California Institute of Technology, 253-37 Caltech, Pasadena, CA 91125

mamikon@caltech.edu

\section{A Sudoku Mystery}

Here is a scrap torn from the corner of a partially completed Sudoku puzzle:

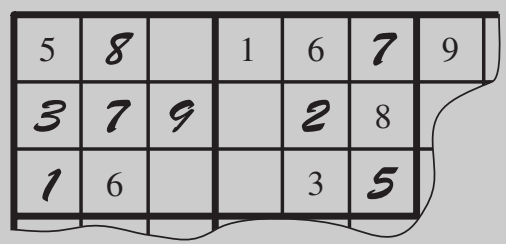

Notice that some of the numbers were printed in the original puzzle, and some were written in by the person who was solving the puzzle before the scrap was torn out. Can you fill in the four empty squares?

(Solution on p. 190) 